\title{
Epidemiology of Parasitic Infections in the West Bank and Gaza Strip, Palestine
}

\author{
Omar Hamarsheh ${ }^{1 *}$ and Ahmad Amro ${ }^{2}$ \\ ${ }^{1}$ Department of Life Sciences, Faculty of Science and Technology, Al-Quds University, Jerusalem, Palestine; \\ ${ }^{2}$ Faculty of Pharmacy, Al-Quds University, Jerusalem, Palestine
}

\begin{abstract}
Parasitic infections are a public health problem that affects all population levels in developing countries including Palestine. The current study describes the epidemiology of parasitic infections in Palestine from 2008 to 2017. Twelve prominent parasitic infections were reported: leishmaniasis (both cutaneous and visceral), malaria, hydatid disease caused by hydatid cysts, toxoplasmosis, ascariasis, oxyuriasis, strongyloidiasis, taeniasis caused by Taenia saginata, amebiasis, giardiasis, and scabies. A total of 137,106 cases were reported; among them, $92,494(67 \%)$ in the Gaza Strip and $44,612(33 \%)$ in the West Bank. This study provides baseline information for better understanding the status of parasitic infections in Palestine and compares their epidemiology between the West Bank and Gaza Strip. Appropriate control measures and health education directed to the public about preventive measures should be considered to raise awareness about parasitic infections to lower their incidences and burden in Palestine.
\end{abstract}

\section{INTRODUCTION}

Parasitic infections are a major public health concern in developing countries. Displacement of people due to conflicts and wars, international travel, and shifting patterns of immigration has increased the importance of raising awareness of these infections. The WHO estimated the global burden of parasitic infections at approximately three billion reported cases and one million deaths per year. ${ }^{1-3}$

Because of their varied life cycles, parasitic infections are known to be associated with geographic and socioeconomic factors, as well as natural disasters and wars, poverty, malnutrition, personal and community hygiene, high population density, unavailability of clean water, low health status, and absence of or poor sanitation. All these conditions enhance the growth, transmission, and dissemination of parasites and increase disease incidence. ${ }^{4-6}$

In Palestine, like other developing countries, parasitic infections are considered a neglected health problem, and they are among the leading causes of morbidity in Palestinian society. According to the Palestinian Ministry of Health, thousands of Palestinians have been exposed to parasites and thousands of them become ill from these infections. ${ }^{7,8}$

In the Gaza Strip, intestinal parasitic diseases have been endemic for a long time; the most common infections include nematodes (Ascaris lumbricoides, Enterobius vermicularis, Strongyloides stercoralis, and Trichuris trichiura), cestodes (Hymenolepis nana), and protozoa (Entamoeba histolytical dispar, Giardia lamblia, and Cryptosporidium parvum). ${ }^{9}$

These pathogens can cause potentially serious diseases and may be fatal if left untreated, especially in children and the elderly.

In the West Bank, few studies have been carried out on gastrointestinal parasites, and no countrywide comprehensive studies on the most common parasitic infections have been conducted so far. ${ }^{9-14}$ Intestinal parasitic infection is still a problem in Palestine, probably because of weak health status, poor sanitation, population crowdedness, mainly in Palestinian refugee camps, bad hygienic habits, and poor health awareness. The main objective of this study was to

* Address correspondence to Omar Hamarsheh, Department of Biological Sciences, Faculty of Science and Technology, P.O. Box 51000, Jerusalem 51000, Palestine. E-mail: ohamarsheh@staff.alquds.edu investigate the epidemiology of parasitic infections reported by the Palestinian Ministry of Health between 2008 and 2017 in the West Bank and Gaza Strip.

\section{MATERIALS AND METHODS}

Study area. Palestine is located in the Middle East with two unconnected geographical areas, the West Bank and Gaza Strip. The West Bank consists of the following districts: Hebron, South Hebron, Bethlehem, Jerusalem, Ramallah, Jericho, Nablus, Jenin, Tubas, Tulkarem, Qalqilya, and Salfit. The Gaza Strip has the following districts: North Gaza, Gaza City, Deir Al Balah, Khan Younis, and Rafah (see Figure 1). The total Palestinian population in 2017 was 4.95 million (three million in the West Bank and 1.95 million in Gaza Strip). ${ }^{15}$ More than $66.2 \%$ of the Palestinian population in the Gaza Strip and $26.6 \%$ of the population in the West Bank are refugees living in crowded camps.

Data collection and analysis. Because many parasitic infections are reportable diseases, the Palestinian Ministry of Health annual statistical reports were screened for the years 2008 until 2017 (the latest published report). Information about the following diseases was collected: leishmaniasis, malaria, hydatid disease, toxoplasmosis, ascariasis, oxyuriasis, strongyloidiasis, taeniasis, amebiasis, giardiasis, and scabies. The data collected included the type of parasitic infection, number of cases, district, and date of infection.

For data analysis, the number of patients for each parasitic disease per year in each Palestinian district was determined. The average annual incidence rate/100,000 inhabitants was calculated for each district.

\section{RESULTS}

There were 12 prominent parasitic infections reported from 2008 to 2017 in Palestine: leishmaniasis (both cutaneous and visceral), malaria, hydatid disease caused by hydatid cysts, toxoplasmosis, ascariasis, oxyuriasis, strongyloidiasis, taeniasis caused by Taenia saginata, amebiasis, giardiasis, and scabies. The geographic distribution of cases in each district in the West Bank and Gaza Strip shows Gaza City, Nablus, North Gaza, Rafah, and Khan Younis with the highest number of cases, and Hebron with the lowest (Table 1, Figure 1). The highest number of reported cases of parasitic infections was 


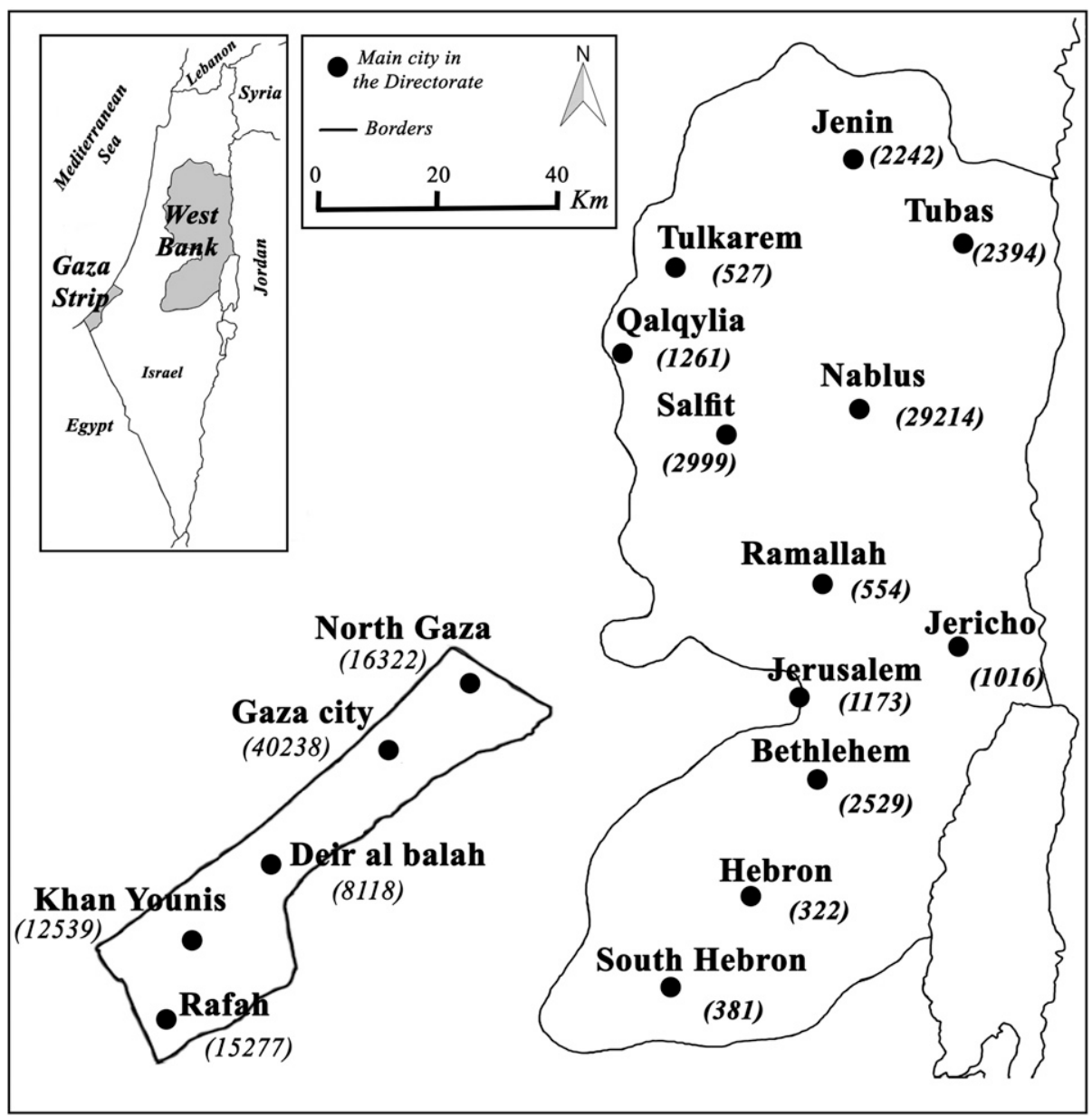

FIGURE 1. Map of the West Bank and Gaza Strip with geographical distribution of parasitic infections in each district with corresponding total number of cases from 2008 to 2017.

in 2008: 25,524 total cases, with 15,337 in the West Bank and 10,187 in the Gaza Strip.

The major parasitic infections reported were those caused by intestinal protozoans, with amebiasis caused by E. histolytica being the most common with 69,771 cases $(6,638$ in the West Bank and 63,133 in the Gaza Strip), followed by giardiasis with 23,062 cases (West Bank 61 and Gaza 22,801), strongyloidiasis with 729 cases (West Bank 667 and Gaza 261), and ascariasis

TABLE 1

Total numbers of cases in Palestine from 2008 to 2017 as reported by Palestinian Ministry of Health

\begin{tabular}{|c|c|c|c|c|c|c|c|c|c|c|c|c|}
\hline & District & 2008 & 2009 & 2010 & 2011 & 2012 & 2013 & 2014 & 2015 & 2016 & 2017 & Total \\
\hline \multirow[t]{6}{*}{ Gaza } & North Gaza & 1,608 & 1,402 & 2,189 & 1,146 & 1,961 & 1,713 & 2,473 & 1,308 & 1,424 & 1,098 & 16,322 \\
\hline & Gaza City & 4,220 & 3,435 & 4,790 & 2,582 & 5,517 & 4,344 & 4,664 & 3,198 & 3,723 & 3,765 & 40,238 \\
\hline & Deir Al Balah & 1,240 & 1,039 & 1,046 & 614 & 1,207 & 662 & 974 & 470 & 426 & 440 & 8,118 \\
\hline & Khan Younis & 1,425 & 861 & 1,219 & 691 & 1,418 & 1,293 & 2,101 & 1,296 & 1,253 & 982 & 12,539 \\
\hline & Rafah & 1,694 & 1,780 & 1,681 & 935 & 1,947 & 1,939 & 1,928 & 1,193 & 1,139 & 1,041 & 15,277 \\
\hline & Total & 10,187 & 8,517 & 10,925 & 5,968 & 12,050 & 9,951 & 12,140 & 7,465 & 7,965 & 7,326 & 92,494 \\
\hline \multirow[t]{14}{*}{ West Bank } & Hebron & 80 & 14 & 22 & 8 & 11 & 10 & 36 & 57 & 54 & 30 & 322 \\
\hline & South Hebron & & 38 & 28 & 11 & 64 & 49 & 60 & 28 & 40 & 63 & 381 \\
\hline & Bethlehem & 115 & 335 & 239 & 129 & 332 & 380 & 309 & 337 & 227 & 126 & 2,529 \\
\hline & Jerusalem & 106 & 83 & 92 & 43 & 186 & 173 & 181 & 121 & 110 & 78 & 1,173 \\
\hline & Ramallah & 4 & 60 & 84 & 48 & 122 & 49 & 64 & 43 & 55 & 25 & 554 \\
\hline & Jericho & 65 & 126 & 123 & 61 & 91 & 53 & 107 & 221 & 112 & 57 & 1,016 \\
\hline & Nablus & 14,069 & 2,270 & 2,246 & 722 & 2,228 & 2,497 & 2,207 & 1,206 & 1,073 & 696 & 29,214 \\
\hline & Jenin & 148 & 1,092 & 234 & 121 & 155 & 113 & 43 & 249 & 26 & 61 & 2,242 \\
\hline & Tulkarem & 29 & 63 & 28 & 27 & 75 & 58 & 99 & 56 & 48 & 44 & 527 \\
\hline & Qalqilya & 49 & 219 & 89 & 30 & 69 & 179 & 136 & 150 & 137 & 203 & 1,261 \\
\hline & Salfit & 672 & 402 & 272 & 67 & 61 & 384 & 377 & 270 & 237 & 257 & 2,999 \\
\hline & Tubas & & 54 & 45 & 50 & 321 & 571 & 498 & 385 & 245 & 225 & 2,394 \\
\hline & Total & 15,337 & 4,756 & 3,502 & 1,317 & 3,715 & 4,516 & 4,117 & 3,123 & 2,364 & 1,865 & 44,612 \\
\hline & Total Palestine & 25,524 & 13,273 & 14,427 & 7,285 & 15,765 & 14,467 & 16,257 & 10,588 & 10,329 & 9,191 & 137,106 \\
\hline
\end{tabular}


with 3,465 cases (West Bank 1,947 and Gaza 1,518). Other parasitic infections reported were oxyuriasis with 28,245 cases (West Bank 27,924 and Gaza 321), leishmaniasis (reported in the West Bank only) with 2,712 cases (cutaneous: 2,672 and visceral: 40), scabies with 8,665 cases (West Bank 4,027 and Gaza 4,638), hydatid disease (in the West Bank only) with 401 cases, and toxoplasmosis with 47 cases (West Bank 27 and Gaza 20). Uncommon parasitic infections reported in the West Bank were seven malaria and two taeniasis ( $T$. saginata) cases.

The yearly trend of the major parasitic infections in the studied period was similar in every year, except for oxyuriasis which had a remarkable peak in 2008 (Figure 2).

The total number of cases for amebiasis and giardiasis fluctuated, with the lowest number of reported cases in 2011 (4,453 and 8,227 cases, respectively), and the highest in 2010 $(8,227$ and 3,080 cases, respectively).

The lowest average annual incidence rate was found for taeniasis (0.01 cases $/ 100,000$ inhabitants in the West Bank). High average annual incidence rates were found for amebiasis (164 cases per 100,000 inhabitants), oxyuriasis (69.8 cases per 100,000 inhabitants), and giardiasis (54.9 cases per 100,000 inhabitants). The average annual incidence rate for taeniasis, toxoplasmosis, hydatid disease, strongyloidiasis, ascariasis, and scabies were $0.01,0.12$, $0.93,1.7,8.3$, and 20.1 cases $/ 100,000$ inhabitants, respectively (Table 2 ).

\section{DISCUSSION}

This study investigated the epidemiology of parasitic infections in Palestine and compared the infection rate between the West Bank and Gaza Strip. The study was based on inspecting health records of the Palestinian Ministry of Health, including patients seeking treatment and referred to health centers and hospitals in all districts in the West Bank and Gaza
Strip with standard and approved diagnostic methods for each parasitic disease.

Parasitic infections caused by pathogenic parasites are common in many developing countries, primarily in children. ${ }^{16-19}$ The existence of these diseases in Palestine is not surprising; most of the cases were reported in the Gaza Strip. Previous reports from Palestine confirmed high levels of infection of intestinal parasites in different regions, especially among school children. ${ }^{6,9,10,12,13}$

The results reported in this study are consistent with other reports about the existence of various parasitic infections in Palestine, especially scabies ${ }^{20}$ and intestinal parasites. ${ }^{9,10,21}$ Oxyuriasis caused by $E$. vermicularis was the most prevalent infection in Nablus, which is a highly populated district with three crowded refugee camps, where most of the cases were reported. Amebiasis and giardiasis were most prevalent in the Gaza Strip. This might be due to the Gaza War that took place in 2008 between Israel and Palestine. During this war, inhabitants especially in Gaza City were forced to leave their homes and settle in crowded temporary camps, where reporting and surveillance of parasitic diseases were severely affected. Moreover, all services, including primary health care, water and food supplies, sanitation, and waste collection services, were either collapsed or completely stopped. These conditions contributed to the increased number of cases during and after the war in $2008 .^{22}$

In the last 10 years, the total number of infected people in Palestine was 137,106 (92,494 (67\%) in Gaza Strip and 44,612 $(33 \%)$ in the West Bank). This variation between the two separated Palestinian regions is related to many factors, including level of poverty, personal hygiene, availability of clean water, functioning sanitary systems, and availability of electricity, all of which are influenced by the repeated wars and continuous siege on the Gaza Strip. The consequences of these factors on public health are very high: $70 \%$ of inhabitants of the Gaza Strip are living below the poverty level in

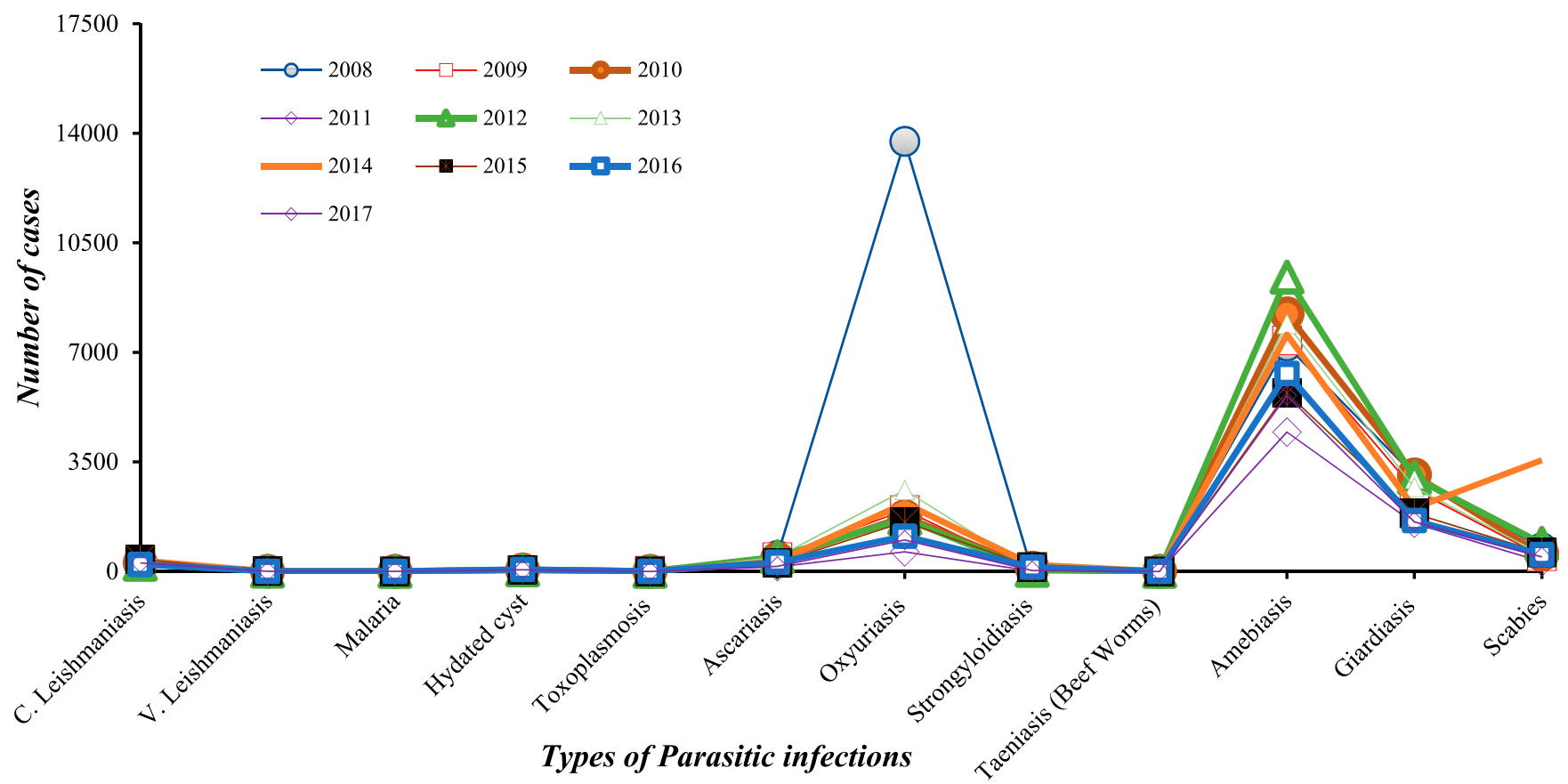

FIGURE 2. Yearly trends of all parasitic infections in Palestine from 2008 to 2017 represented by the number of cases. This figure appears in color at www.ajtmh.org. 
TABLE 2

Total number of cases and average annual incidence rate/100,000 inhabitants for each parasitic disease in the West Bank and Gaza Strip districts

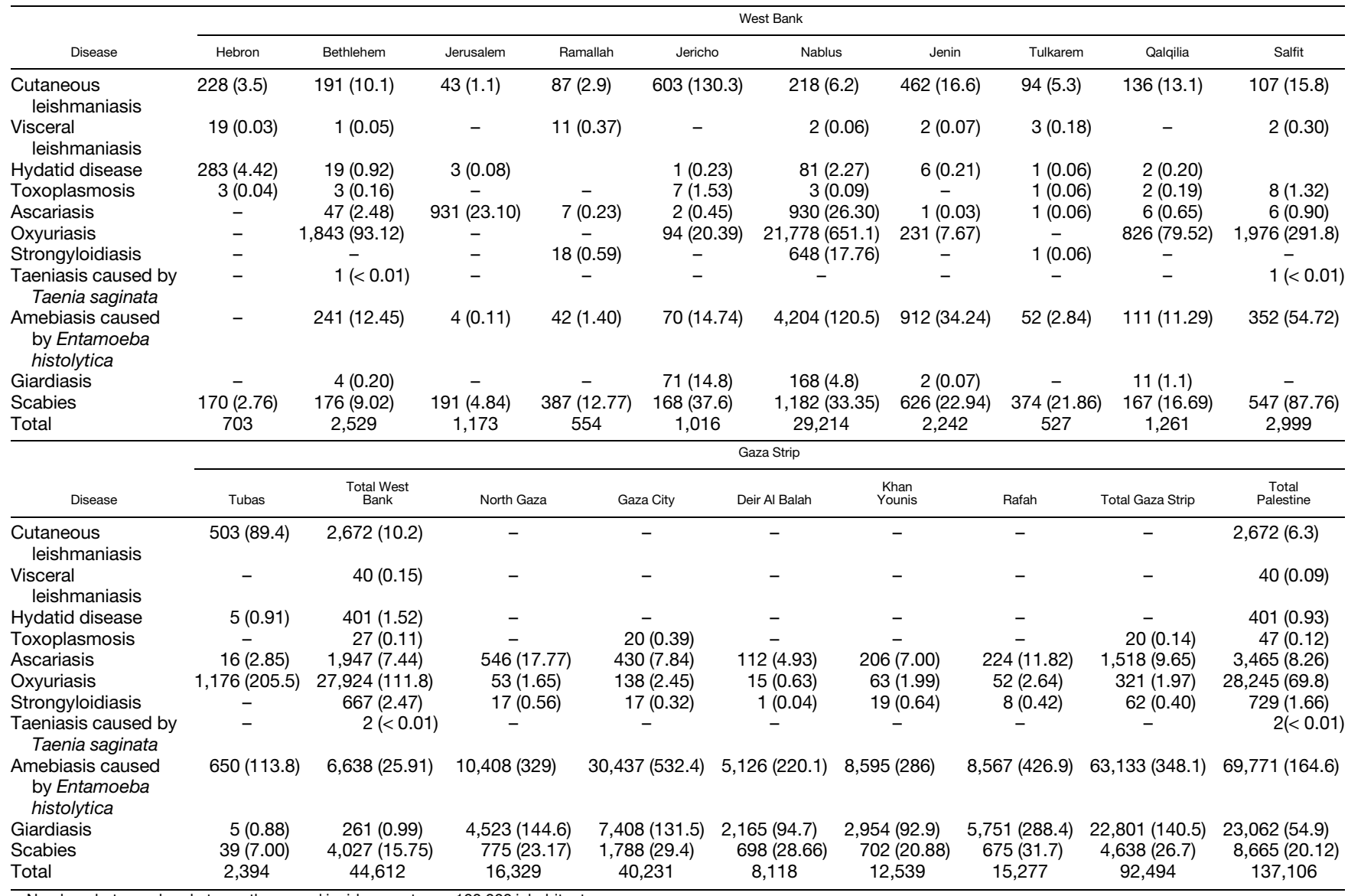

Numbers between brackets are the annual incidence rate per 100,000 inhabitants.

highly crowded areas. More than $90 \%$ of people living in the Gaza Strip do not have access to clean drinking water, and most natural water resources are contaminated. However, Palestinians living in the West Bank have better access to health services, clean drinking water, and functioning sanitary sewer systems which contribute to lower disease prevalence.

The number of reported toxoplasmosis cases was surprisingly very low in both the West Bank and Gaza Strip (27 and 20 cases). Serological diagnosis of toxoplasmosis during pregnancy is not mandatory and carried out on request. Two studies on seroprevalence of toxoplasmosis among Palestinian women who are pregnant or received an abortion in Hebron in the West Bank ${ }^{23}$ and Gaza City ${ }^{24}$ in Gaza Strip showed high seroprevalence and recommended routine lgG and IgM tests in early stages of pregnancy to reduce the effects of the disease on mothers and babies. Health awareness programs were routinely conducted by the Child and Mother Health Unit at the Palestinian Ministry of Health to advise pregnant women to avoid contact with domestic cats. On the other hand, scabies infection was highly prevalent in both the West Bank and Gaza Strip. Recent studies on epidemiology of scabies in Palestine have identified several risk factors associated with the disease. These include crowding, poor hygiene, and low socioeconomic status. ${ }^{20,25}$

Leishmaniasis is endemic in the West Bank, but no cases were reported in the Gaza Strip. Cutaneous leishmaniasis infection in the West Bank comprised about $1.9 \%$ of the total parasitic infections in Palestine, with Leishmania tropica and Leishmania major as the main causative agents. Leishmaniasis is prevalent in regions with high agricultural activities such as Jericho, Jenin, and Tubas. ${ }^{26}$ Visceral leishmaniasis is caused by Leishmania infantum which is prevalent in the western parts of the West Bank, extending to central Israel. ${ }^{27}$

No leishmaniasis cases were reported in the Gaza Strip, probably because of unfavorable conditions for the sand fly vectors or the Leishmania parasites, although cutaneous leishmaniasis is endemic in the nearby Sinai and Negev deserts. $^{28}$

Malaria was completely eradicated in Palestine between 1922 and $1925 .^{29}$ The seven reported cases in this study were imported, and patients reported traveling to malaria-endemic areas before their infection. Thus, local transmission of the parasite is very unlikely in Palestine.

Strongyloidiasis and ascariasis were reported in both the Gaza Strip and West Bank, with the highest numbers reported in Nablus. A household survey in the Gaza Strip documented that $56 \%$ of examined individuals were infected with Ascaris lumbricoides and $15.5 \%$ with S. stercoralis. A similar infection trend was found in the northern districts of the West Bank. Both infections were associated with age, level of education, and socioeconomic conditions. ${ }^{12,30}$

Limitations were identified in this study. First, data were collected from annual health reports of the Palestinian Ministry of Health, which could have underestimated the prevalence 
because of underreporting and limitations in laboratory diagnosis. Second, many of the diagnoses were based on voluntary reporting by health departments, likely leading to underestimation. Third, information on seasonality of the infections was not evaluated. Fourth, sociodemographic information could not be collected.

Further research is needed to guide public health and clinical recommendations to control parasitic infections. Community health education campaigns directed to the public and health care professionals concerning preventive measures would help raise awareness about parasitic infections. Local health authorities should provide routine observation of food handlers as well as the level of personal hygiene of employees in restaurants, schools, and hospitals. Because of frequent electricity interruptions in the Gaza Strip, untreated sewage is pumped directly to the sea or open pools, directly contributing to the high number of intestinal parasite infections.

Prevention and control measures should be directed to increase awareness toward these diseases. Meanwhile, other parasitic diseases exist in Palestine but were not reported by the Palestinian Ministry of Health. Therefore, current surveillance programs should be thoroughly revised and evaluated.

Received July 23, 2019. Accepted for publication October 14, 2019. Published online November 25, 2019.

Acknowledgments: We would like to thank Dalia Nassar, Palestinian Ministry of Health, and Zamallah Academic Development Program sponsored by Taawon Association. We sincerely thank Catherine Flanley and Hannah Corman for editing and correcting language errors.

Financial support: Funding in the form of fellowships was provided to O. H. and A. A. by Zamallah Academic Development Program sponsored by Taawon Association.

Authors' addresses: Omar Hamarsheh, Department of Life Sciences, Faculty of Science and Technology, Al-Quds University, Jerusalem, Palestine, E-mail: ohamarsheh@staff.alquds.edu. Ahmad Amro, Faculty of Pharmacy, Al-Quds University, Jerusalem, Palestine, E-mail: ahmad.amro@staff.alquds.edu.

\section{REFERENCES}

1. Torgerson PR et al., 2015. World Health Organization estimates of the global and regional disease burden of 11 foodborne parasitic diseases, 2010: a data synthesis. PLoS Med 12: e1001920.

2. Pisarski K, 2019. The global burden of disease of zoonotic parasitic diseases: top 5 contenders for priority consideration. Trop Med Infect Dis 4: 2-9.

3. Piperaki ET, Tassios PT, 2016. Parasitic infections: their position and impact in the postindustrial world. Clin Microbiol Infect 22: 469-470.

4. Karan A, Chapman GB, Galvani A, 2012. The influence of poverty and culture on the transmission of parasitic infections in rural nicaraguan villages. J Parasitol Res 2012: 478292.

5. Smith Darr J, Conn DB, 2015. Importation and transmission of parasitic and other infectious diseases associated with international adoptees and refugees immigrating into the United States of America. Biomed Res Int 2015: 763715.

6. Leiby DA et al., 2019. International survey on the impact of parasitic infections: frequency of transmission and current mitigation strategies. Vox Sang 114: 17-27.

7. PMoH, 2017. Health Annual Report. Nablus, Palestine: Palestinian Health Information Center.

8. Hamarsheh O, Nasereddin A, Damaj S, Sawalha S, Al-Jawabreh H, Azmi K, Amro A, Ereqat S, Abdeen Z, Al-Jawabreh A, 2012. Serological and molecular survey of Leishmania parasites in apparently healthy dogs in the West Bank, Palestine. Parasit Vectors 5: 183

9. Alhindi AlH, Mervat A-L, 2013. Trends of intestinal parasites prevalence in the Gaza Strip, 1998-2007: the use of government health records. Turk J Med Sci 43: 652-659.

10. Al-Hindi Al, El-Kichaoi AY, 2015. Occurrence of gastrointestinal parasites among pre- school children, Gaza, Palestine. IUGNS 16: $125-130$.

11. Mezeid N, Shaldoum F, Al-Hindi Al, Mohamed FS, Darwish ZE, 2014. Prevalence of intestinal parasites among the population of the Gaza Strip, Palestine. Ann Parasitol 60: 281-289.

12. Hussein AS, 2011. Prevalence of intestinal parasites among school children in northern districts of West Bank-Palestine. Trop Med Int Health 16: 240-244.

13. Bdir S, Adwan G, 2010. Prevalence of intestinal parasitic infections in Jenin Governorate, Palestine: a 10-year retrospective study. Asian Pac J Trop Med 3: 745-747.

14. Abdelateef N, Elmanama AA, 2010. Bacterial and Parasitic Etiologic Agents among Acute Gastroenteritis Patients in Gaza Strip, Palestine. Available at: http://hdl.handle.net/20.500.12358/ 25352. Accessed July 2019.

15. PCBS, 2018. Annual Report 2017, Palestinian Central Bureau of Statistics. Ramallah, State of Palestine.

16. WHO, 2010. Working to Overcome the Global Impact of Neglected Tropical Diseases: First WHO Report on Neglected Tropical Diseases. Geneva, Switzerland: World Health Organization.

17. Akdur Ozturk E, Unver A, 2017. Travel-related parasitic infections in travellers to southeast Asia and Western Pacific countries. Turkiye Parazitol Derg 41: 239-245.

18. Amer OSO, Al-Malki ES, Waly MI, AlAgeel A, Lubbad MY, 2018. Prevalence of intestinal parasitic infections among patients of king fahd medical city in riyadh region, Saudi arabia: a 5-year retrospective study. J Parasitol Res 2018: 8076274.

19. Bahk YY, Shin EH, Cho SH, Ju JW, Chai JY, Kim TS, 2018. Prevention and control strategies for parasitic infections in the Korea centers for disease control and prevention. Korean $J$ Parasitol 56: 401-408.

20. Amro A, Hamarsheh O, 2012. Epidemiology of scabies in the west bank, Palestinian territories (occupied). Int J Infect Dis 16: e117-e120.

21. Al-Shawa R, 2006. Intestinal parasites infections in refugees camps in the Gaza governorates, Palestine. Internet J Parasitic Dis 1: 1-6.

22. Radi S, El Sayed N, Nofal L, Abdeen Z, 2013. Ongoing deterioration of the nutritional status of Palestinian preschool children in Gaza under the Israeli siege. East Mediterr Health $J$ 19: 234-241.

23. Nijem KI, Al-Amleh S, 2009. Seroprevalence and associated risk factors of toxoplasmosis in pregnant women in Hebron district, Palestine. East Mediterr Health J 15: 1278-1284.

24. Al-Hindi Al, Lubbad AMM, 2009. Seroprevalence of toxoplasmosis among Palestinian aborted women in Gaza. Ann Alaqsa Med 5: 39-47.

25. Almomani T, 2014. Scabies in children in sector Gaza. Middle East $J$ Fam Med 99: 1-4.

26. Sawalha SS, Shtayeh MS, Khanfar HM, Warburg A, Abdeen ZA, 2003. Phlebotomine sand flies (Diptera: psychodidae) of the Palestinian West Bank: potential vectors of leishmaniasis. $J$ Med Entomol 40: 321-328.

27. Amro A, Azmi K, Schonian G, Nasereddin A, Alsharabati MB, Sawalha S, Hamarsheh O, Ereqat S, Abdeen Z, 2009. Epidemiology of paediatric visceral leishmaniasis in Hebron district, Palestine. Trans $R$ Soc Trop Med Hyg 103: 731.

28. Jaffe CL, Baneth G, Abdeen ZA, Schlein Y, Warburg A, 2004. Leishmaniasis in Israel and the Palestinian authority. Trends Parasitol 20: 328-332.

29. Kligler I, 1925. Report on Sanitation of the Upper Jordan Valley. Malaria Commission Report. Chap. 52. Geneva, Switzerland: World Health Organization.

30. Al-Zain B, Al-Hindi Al, 2005. Distribution of Strongyloides stercoralis and other intestinal parasites in household in Beit-lahia city, Gaza Strip, Palestine. Ann Alquds Med 1: 48-52. 